\title{
Neck spasm after chemoradiotherapy for head and neck cancer: Natural history and dosimetric correlates
}

\author{
Klaudia U. Hunter, MD, ${ }^{1 *}$ Francis Worden, MD, ${ }^{2}$ Carol Bradford, MD, ${ }^{3}$ Mark Prince, MD, ${ }^{3}$ Scott McLean, MD, ${ }^{3}$ Gregory Wolf, MD, ${ }^{3}$ Douglas B. Chepeha, MD, \\ $\mathrm{MPH},{ }^{3}$ Avraham Eisbruch, $\mathrm{MD}^{1}$ \\ ${ }^{1}$ Department of Radiation Oncology, University of Michigan, Ann Arbor, Michigan, ${ }^{2}$ Department Hematology-Oncology, University of Michigan, Ann Arbor, Michigan, ${ }^{3}$ Department \\ Otolaryngology-Head Neck Surgery, University of Michigan, Ann Arbor, Michigan.
}

Accepted 23 January 2013

Published online 4 April 2013 in Wiley Online Library (wileyonlinelibrary.com). DOI 10.1002/hed.23284

\begin{abstract}
Background. Little is known about the determinants of postradiation neck spasms in patients with head and neck cancer.

Methods. Patients with head and neck cancer treated with radiation therapy (RT) from 2004 to 2010 who experienced neck spasms were reviewed. Radiation doses were generated for their sternocleidomastoid (SCM) muscles bilaterally. Unaffected SCMs were used as controls.

Results. Thirty-four patients reported neck spasms. Thirty had received definitive chemoradiation, and 4 had RT alone. Seven also had an ipsilateral neck dissection. Median time to onset was 23 months (range, 6-67 months). There were significantly higher radiation doses to the
\end{abstract}

affected SCMs with a median of the mean dose to the affected and unaffected SCM of 62.3 Gy (range, 29-71 Gy) and 53.7 Gy (range, 27-65 Gy), respectively $(p<.0001)$. Other dosimetric variables were also statistically significant but were highly correlated with the mean SCM dose. Neck dissection did not affect our results.

Conclusion. Neck spasms after chemotherapy intensity-modulated radiation therapy (IMRT) shows a strong dose-response relationship. (C) 2013 Wiley Periodicals, Inc. Head Neck 36: 176-180, 2014

KEY WORDS: head and neck cancer, neck spasms, complications, myoclonus, radiation

\section{INTRODUCTION}

As tumor control rates and treatment outcomes improve, there has been increasing interest in minimizing treatment-related toxicity for patients with head and neck cancer. Although it is common to have an assortment of vague, brief, uncomfortable sensations in the area of previous treatment, we have seen a consistent trend of neck muscle spasm symptoms late after therapy, described as a "Charlie horse-like", sensation by our patients. Very little has been published in the medical literature about this late effect. In this study, we report the clinical details of this complication and its dosimetric correlates.

\section{MATERIALS AND METHODS}

As part of our routine clinical practice, we identified and tracked all patients complaining of neck spasms after radiation therapy at the University of Michigan between 2004 and 2010. After these complaints were first noticed, we have documented the frequency, quality, and duration of symptoms, as well as any triggers, alleviating or aggravating factors, and treatments received for this complication. Routine clinical follow-up after therapy consists of

*Corresponding author: K. U. Hunter, University of Michigan, Department of Radiation Oncology, 1500 E. Medical Center Dr., UH B1C490 SPC 5010, Ann Arbor, Ml 48109. E-mail: kurbania@med.umich.edu visits every 2 months during the first 2 years, every 3 months in the third year, and every 6 months thereafter through 5 years. We have now retrospectively reviewed these records to collect this data, as well as clinical and treatment variables. This retrospective study was approved by the Institutional Review Board of the University of Michigan with a waiver of informed consent.

The sternocleidomastoid (SCM) muscles for these patients were contoured using our in-house treatment planning system, UMPlan. The entire length of the SCM muscles was contoured from its origin at the manubrium and clavicle to its insertion at the mastoid and occipital bone bilaterally. Dose-volume histograms were calculated separately for the SCM on each side of the neck. For each patient, an "affected" and "unaffected" side of the neck was determined from the clinical follow-up notes.

Statistical analysis was performed using a $t$ test comparing dosimetric variables for the affected and unaffected SCM muscles in each patient. For patients with unilateral neck spasms, the unaffected side was used as an internal control. For patients with bilateral neck spasms, both sides were included in the analysis as affected SCM muscles. Pearson's correlation coefficient was used to evaluate the association between dosimetric factors and occurrence of spasm.

In addition to the above analysis, a second comparison was performed in an attempt to elucidate clinical factors related to the development of neck spasms. Twelve of the patients identified with neck spasms (as described above) 
were treated on a prospective clinical protocol $(n=72)$ for patients with stage III to IV oropharyngeal cancer. These patients received definitive chemoradiation with weekly carboplatin (area under the curve =1) and paclitaxel $\left(30 \mathrm{mg} / \mathrm{m}^{2}\right)$. Radiation was delivered using intensity-modulated radiation therapy (IMRT) with a prescription dose of 70 Gy to the primary tumor and involved lymph nodes, 59 to 63 Gy to high-risk nodal regions and the anatomic compartments around the gross tumor volumes, and 56 to 59 Gy to low-risk nodal regions. Priority was given during planning to spare the major salivary glands, pharyngeal constrictor muscles, larynx (glottis and supraglottic larynx), and esophagus, to minimize late xerostomia and dysphagia. Details of the radiation treatment planning have been previously published. ${ }^{1}$

The clinical variables from the 12 protocol patients who developed neck spasms were compared with the 60 protocol patients who did not develop neck spasms. A 1-way analysis of variance test was used for this comparison.

\section{RESULTS}

Thirty-four patients developed posttreatment neck spasms. Their median age was 58 years (range, 42-71 years). There were 31 men and 3 women. The primary site of disease was unknown in 4 patients, glottic larynx in 1 patient, supraglottic larynx in 2 patients, retromolar trigone in 1 patient, and oropharyngeal in 26 patients (15 patients with tonsil, 10 patients with base of tongue, and 1 patient with a posterior pharyngeal wall cancer). During this period of time, we treated 221 patients with oropharyngeal cancer (12\% developed neck spasms), 28 patients with unknown primary cancer (14\% developed neck spasms), 33 patients with laryngeal cancer (3\% developed neck spasms), 50 patients with supraglottic cancer (4\% developed neck spasms), and 20 patients with oral cavity cancer (5\% developed neck spasms).

Thirty patients $(88 \%)$ received definitive concurrent chemoradiation therapy, 2 patients received definitive radiation alone, and 2 patients received postoperative radiation alone. Seven patients underwent neck dissection on the affected side before the onset of their neck spasms (3 had surgery preradiation therapy and 4 had surgery postradiation therapy).

The median follow-up is 51 months (range, 30-90 months) with most patients continuing their follow-up at our department, at the time of this publication. Twenty-six patients developed unilateral neck spasms and 8 had bilateral neck symptoms. In all cases of unilateral symptoms, the affected neck side was ipsilateral to the site of the primary tumor (in patients with lateralized tumors). The median time to symptom onset was 23 months (range, 6-67 months). Once symptoms began, the median duration of symptoms was 24 months (range, 2-48 months) with all but 6 patients continuing to have symptoms at the time of their last follow-up.

Patients describe their neck spasms as a "Charlie horse-like" painful contraction in their neck, typically pointing to the SCM as the site of spasm. These myoclonus events are brief and last less than 1 minute in all but 1 patient who described events lasting 4 to 5 minutes. Spasm events were triggered by specific neck positions and movements such as head turning, yawning, and strenuous physical activity such as weight lifting. Spasms are commonly alleviated by neck stretching or massage. For most patients, these neck spasms were not painful. However, 7 patients did require additional intervention because these spasms were painful and/or distressing. Two patients were referred for physical therapy, and 6 patients required some form of medication (potassium supplements in 1 patient, aspirin in 1 patient, benzodiazepines or muscle relaxers in 3 patients, and gabapentin and tramadol in 1 patient). Only 1 patient reported that neck spasms necessitated a leave of absence from work (this patient previously worked as a truck driver and reported that turning his neck while driving caused him significant pain). Two additional patients complained that SCM muscle twitching caused them embarrassment at work but did not interfere with their ability to perform their jobs.

In addition, the severity of the neck spasms waxed and waned over time with abrupt exacerbations occasionally occurring long after the end of radiation therapy (RT). For 1 patient, symptoms were mild until they suddenly worsened after a strenuous basketball game 24 months after the completion of his RT. At his last follow-up, he reported that his symptoms were still continuing at the increased level of severity.

Physical examination of the neck was unremarkable in all patients, and no patients had evidence of muscle, skin, or soft tissue fibrosis or limitation in neck movements.

Dosimetric data compiled from the affected and unaffected SCM muscles is listed in Table 1. The medians of

TABLE 1. Dosimetric data for sternocleidomastoid muscles with and without neck spasms.

\begin{tabular}{|c|c|c|c|}
\hline & SCM without neck spasms, median (range) & SCM with neck spasms, median (range) & $p$ value \\
\hline Mean dose & 53.7 Gy (27.3-65.4) & 62.3 Gy (29.4-71.0) & .00001 \\
\hline Max dose to $0.5 \%$ volume (D0.5) & 65.0 Gy (58.4-74.9) & 73.8 Gy $(63.0-77.5)$ & $<.00001$ \\
\hline Max dose to $20 \%$ volume (D20) & 61.4 Gy (55.6-72.4) & 68.7 Gy $(60.7-75.3)$ & $<.00001$ \\
\hline Max dose to $95 \%$ volume (D95) & 22.2 Gy $(0.6-56.7)$ & 48.4 Gy $(0.2-60.5)$ & $<.00001$ \\
\hline Max dose to $0.5 \mathrm{cc}(\mathrm{D} 0.5 \mathrm{cc})$ & 64.8 Gy $(58.3-74.5)$ & 73.3 Gy $(62.9-77.4)$ & $<.00001$ \\
\hline Max dose to $1.0 \mathrm{cc}$ (D1 cc) & 64.1 Gy $(58.0-73.9)$ & 72.6 Gy $(62.6-77.3)$ & $<.00001$ \\
\hline Volume receiving $\geq 40$ Gy (V40) & $86.2 \%(40.0-100.0)$ & $98.2 \%(43.3-100)$ & .0002 \\
\hline Volume receiving $\geq 50$ Gy (V50) & $78.9 \%(31.8-98.7)$ & $94.6 \%(39.7-99.7)$ & .00002 \\
\hline Volume receiving $\geq 60$ Gy (V60) & $43.5 \%(0-89.4)$ & $77.5 \%$ (28.4-95.8) & $<.00001$ \\
\hline Volume receiving $\geq 65$ Gy (V65) & $0.5 \%(0-69.1)$ & $46.3 \%(0-86.9)$ & $<.00001$ \\
\hline Volume receiving $\geq 70$ Gy (V70) & $0 \%(0-53.9)$ & $10.1 \%(0-76.02)$ & .003 \\
\hline
\end{tabular}

Abbreviations: SCM, sternocleidomastoid; max, maximum. 


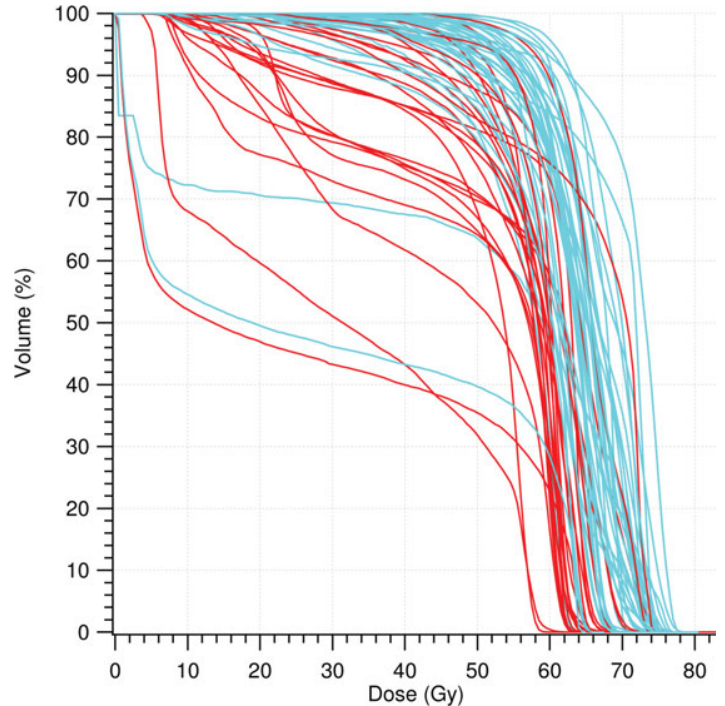

FIGURE 1. Higher overall radiation dose to sternocleidomastoid muscles affected by posttreatment neck spasms. Cumulative dose volume histograms for sternocleidomastoid muscles on the side of the neck affected (dark red) and unaffected (light blue) by posttreatment neck spasms. [Color figure can be viewed in the online issue, which is available at wileyonlinelibrary.com.]

the mean doses to the SCM for the affected and unaffected muscles were 62.3 Gy (range, 29-71 Gy) and 53.7 Gy (range, 27-65 Gy), respectively $(p<.0001)$. Using the mean dose for all SCM muscles (58 Gy) as a cutoff value had a sensitivity of $83 \%$, specificity of $77 \%$, positive predictive value of $85 \%$, and negative predictive value of $74 \%$ for predicting posttreatment neck spasms. Other dosimetric variables were also statistically significant but were highly correlated with the mean SCM dose (correlation coefficient $\geq 0.76$ for all variables listed in Table 1, except for V70 with a correlation coefficient of 0.57). Cumulative dose volume histograms for all SCM analyzed are shown in Figure 1.

We also examined the utility of using a mean dose of 56 Gy to the SCM as a cutoff value for predicting neck spasms because 56 Gy is used as an elective nodal dose in Radiation Therapy Oncology Group trials. Thirty-one percent of SCM muscles ( 8 of 26) without neck spasms received a mean dose 56 Gy or more compared with $90 \%$ of SCM muscles (38 of 42) with neck spasms. These results suggest that keeping mean SCM doses below 56 Gy is likely to decrease the incidence of this posttreatment complication.

In order to exclude the effect of neck dissection on the occurrence of neck spasms and its potential influence on dose-effect relationships, the same analysis was performed excluding the 7 patients who had undergone a neck dissection as part of their treatment. These results are shown in Table 2, demonstrating very similar doses and statistically significant differences between the SCMs in the affected and nonaffected neck sides as those found in the whole population (Table 1) of patients with symptoms. During the period of this study, 221 patients with oropharyngeal cancer were treated. Twenty-eight percent (62 of 221 patients) underwent neck dissection (only 1 patient underwent neck dissection pretreatment). The rate of neck dissection among patients with oropharyngeal cancer that developed neck spasms (19\%; 5 of 26 patients) was lower than the rate for patients with oropharyngeal cancer that did not develop neck spasms (29\%; 57 of 195 patients). This trend is difficult to interpret as our general rate of posttreatment neck dissection has declined with time as part of our institutional practice.

In order to examine which clinical variables may be related to the development of neck spasms, we analyzed the dataset of 72 patients with oropharyngeal cancer treated with definitive chemo-IMRT as part of a prospective study at our institution. Of these 72 patients, 12 patients had neck spasms and were included in the analysis in this article. Comparison of these 12 patients to the other 60 patients who were treated in an identical manner but did not develop neck spasms (Table 3), showed that the patients with neck spasms had significantly lower $\mathrm{T}$ stage and were more likely to be nonsmokers.

In order to assess whether better prognosis patients lived longer and were therefore more likely to develop neck spasms, we also examined the survival data for this 72 patient cohort of patients with oropharyngeal cancer. All 12 patients in this cohort that developed neck spasms were alive and continuing to follow-up with us (median follow-up 5.8 years; range, 4.6-8.9 years). The 60 patients in this group that did not develop neck spasms were followed for a median of 4.9 years (range, 6-67 months) with 14 deaths in this group. Only 4 patients in

TABLE 2. Dosimetric data for sternocleidomastoid muscles with and without neck spasms, excluding patients undergoing neck dissection.

\begin{tabular}{|c|c|c|c|}
\hline & SCM without neck spasms, median (range) & SCM with neck spasms, median (range) & $p$ value \\
\hline Mean dose & 53.2 Gy (27.8-65.4) & 60.9 Gy (29.4-71.0) & .00009 \\
\hline Max dose to $0.5 \%$ volume (D0.5) & 66.4 Gy $(62.4-74.9)$ & 72.7 Gy $(63.0-77.5)$ & $<.00001$ \\
\hline Max dose to $20 \%$ volume (D20) & 63.1 Gy $(59.7-72.4)$ & 68.2 Gy $(60.7-75.3)$ & $<.00001$ \\
\hline Max dose to $95 \%$ volume (D95) & 27.0 Gy $(0.6-56.7)$ & 43.4 Gy $(0.2-60.5)$ & .0003 \\
\hline Max dose to $0.5 \mathrm{cc}$ (D0.5 cc) & 66.1 Gy $(62.3-74.5)$ & 72.3 Gy $(62.9-77.4)$ & $<.00001$ \\
\hline Max dose to $1.0 \mathrm{cc}$ (D1 cc) & 65.6 Gy $(61.9-73.9)$ & 71.9 Gy (62.6-77.3) & $<.00001$ \\
\hline Volume receiving $\geq 40$ Gy (V40) & $84.1 \%(40.0-100.0)$ & $94.6 \%(43.3-100)$ & .004 \\
\hline Volume receiving $\geq 50$ Gy (V50) & $77.6 \%(35.4-98.7)$ & $90.0 \%(39.7-99.5)$ & .001 \\
\hline Volume receiving $\geq 60$ Gy (V60) & $46.5 \%(16.5-89.4)$ & $72.1 \%(28.4-95.8)$ & .00001 \\
\hline Volume receiving $\geq 65$ Gy (V65) & $11.6 \%(0-69.1)$ & $43.2 \%(0-86.9)$ & $<.00001$ \\
\hline Volume receiving $\geq 70$ Gy (V70) & $4.4 \%(0-53.9)$ & $17.1 \%(0-76.02)$ & .01 \\
\hline
\end{tabular}

Abbreviations: SCM, sternocleidomastoid; max, maximum. 
TABLE 3. Comparison of patients with oropharyngeal cancer treated on the same prospective protocol $(n=72)$.

\begin{tabular}{|c|c|c|c|}
\hline & Patients without neck spasm & Patients with neck spasm & $p$ value \\
\hline Age & Median, 55 y & Median, 52 y & NS \\
\hline \multirow[t]{2}{*}{ Sex } & 6 females $(10 \%)$ & 2 females $(17 \%)$ & NS \\
\hline & 54 males $(90 \%)$ & 10 males $(83 \%)$ & \\
\hline \multirow[t]{4}{*}{ T classification } & T1: 7 patients $(12 \%)$ & T1: 2 patients (17\%) & .02 \\
\hline & T2: 20 patients $(33 \%)$ & T2: 9 patients $(75 \%)$ & \\
\hline & T3: 15 patients $(25 \%)$ & T3: 1 patient $(8 \%)$ & \\
\hline & T4: 18 patients $(30 \%)$ & T4: 0 patients & \\
\hline \multirow[t]{6}{*}{$\mathrm{N}$ classification } & N0: 5 patients $(8 \%)$ & N0: 0 patients & NS \\
\hline & N1: 4 patients $(7 \%)$ & N1: 2 patients (17\%) & \\
\hline & N2a: 7 patients (12\%) & N2a: 1 patient $(8.3 \%)$ & \\
\hline & N2b: 26 patients (43\%) & N2b: 7 patients $(58 \%)$ & \\
\hline & N2c: 13 patients $(22 \%)$ & N2c: 1 patient $(8.3 \%)$ & \\
\hline & N3: 5 patients $(8 \%)$ & N3: 1 patients $(8.3 \%)$ & \\
\hline \multirow[t]{3}{*}{ AJCC stage } & Stage 3: 6 patients $(10 \%)$ & Stage 3: 2 patients $(17 \%)$ & NS \\
\hline & Stage 4a: 49 patients $(82 \%)$ & Stage 4a: 9 patients $(75 \%)$ & \\
\hline & Stage $4 \mathrm{~b}$ : 5 patients $(8 \%)$ & Stage $4 b$ : 1 patient $(8 \%)$ & \\
\hline \multirow[t]{2}{*}{ Disease site } & Tonsil: 28 patients $(47 \%)$ & Tonsil: 6 patients $(50 \%)$ & NS \\
\hline & Base of tongue: 32 patients $(53 \%)$ & Base of tongue: 6 patients $(50 \%)$ & \\
\hline \multirow[t]{3}{*}{ Smoking status } & Current: 16 patients $(27 \%)$ & Current: 0 patients & .005 \\
\hline & Former: 27 patients $(45 \%)$ & Former: 3 patients (25\%) & \\
\hline & Never: 17 patients $(28 \%)$ & Never: 9 patients $(75 \%)$ & \\
\hline
\end{tabular}

Abbreviations: NS, not significant; AJCC, American Joint Committee on Cancer.

the "no spasm group' lived less than 23 months, which is the median time to onset of neck spasms for all patients, suggesting that nearly all patients in both groups lived long enough to be at risk for developing neck spasms.

\section{DISCUSSION}

This series describes myoclonus episodes centered on the SCM starting at a median of 23 months after the completion of therapy. The rate of these episodes has been $12 \%$ to $14 \%$ in patients with oropharyngeal or unknown primary tumors receiving comprehensive chemoradiation at our institution. To our knowledge, only 2 case series have been previously published documenting this phenomenon. $^{2,3}$ Van Daele et $\mathrm{al}^{2}$ reported a series of 9 patients treated with RT to the head and neck region ( 8 patients with head and neck cancer and 1 patient with Merkel cell carcinoma involving the cheek), and the group at Memorial Sloan-Kettering Cancer Center have presented a cohort of 18 patients with this complication. $^{3-6}$ In both of these series, botulinum A toxin has been successfully used to treat pain in refractory patients.

Our series is unique in assessing the prevalence of myoclonus among patients receiving comprehensive chemo-RT, its course over time, and its relationships with the doses delivered to the SCM. In our group, only 7 of 33 patients required any intervention specifically for neck spasms with only 1 patient reporting that spasms interfered with his daily life and required narcotic medication. This complication can continue on for many years and can change unpredictably over time with only 6 of our 33 patients reporting resolution of their symptoms at their last follow-up despite a median follow-up of 4.25 years. Fortunately for most patients, the symptoms were mild, brief, and do not require treatment.
The mechanism of postradiation muscle injury is an ongoing area of investigation, but given its late onset, it is likely related to chronic vascular injury or direct myocyte injury. Animal studies using large single doses of radiation have shown delayed fibrosis and vascular lesions characteristic of radiation injury with the resulting loss of large numbers of muscle fibers years after radiation exposure $(50 \%$ loss of muscle fibers with a single dose of $21 \mathrm{~Gy}) .^{7}$ Animal studies have also shown changes in muscle metabolism with the increased deposition of extracellular collagen potentially disrupting myocyte organization and communication, as well as elevated levels of transforming growth factor-beta in myocytes, inflammatory cells, and extracellular matrix up to a year after RT suggesting a role for cytokines in signaling posttreatment fibrosis. $^{7}$ Examination of human tissue from patients experiencing radiation-induced myopathy also demonstrates muscle atrophy, fibrosis, and radiation-induced vascular injury. ${ }^{8}$ Doses above 63 Gy in standard fractionation have been shown to cause muscle edema, decreased muscle strength, and decreased range of motion in patients treated for soft tissue sarcomas of the extremities. ${ }^{9}$

In this article, we report an $83 \%$ sensitivity and $77 \%$ specificity for a cutoff value of 58 Gy for mean dose in predicting posttreatment neck spasms. This value was chosen because it was the average of the mean dose for all SCM muscles in this patient group. However, many dosimetric factors were highly correlated with the development of posttreatment neck spasms. As these factors were also highly correlated with the mean SCM doses, the mean dose remains as the easiest parameter to use. Further studies would be needed to validate this finding and find the best cutoff dosimetric value.

In the patients with unilateral symptoms, the affected side of the neck was ipsilateral to the site of the primary 
tumor, where nodal disease was more prevalent and, therefore, the prescribed radiation doses were higher. A substantial reduction of the doses to the SCMs is usually not attainable when gross nodal disease is treated. However, if studies of reduced radiation doses for patients with human papillomavirus-related oropharyngeal cancers are successful, reducing the doses to the involved neck in such patients in the future will be expected to reduce the rate of late neck spasms, as demonstrated in the dosimetric comparisons in this article.

The only clinical factors that were imbalanced between patients with oropharyngeal cancer that did and did not develop neck spasms after treatment on a prospective protocol with concurrent treatment were lower $\mathrm{T}$ classification and a higher rate of nonsmokers among patients who developed neck spasms. This could be explained by a higher rate of neck spasms among patients with human papillomavirus-related oropharyngeal cancers because these patients tend to be nonsmokers and have an earlier tumor stage with more-advanced nodal disease.

The strength of this study is its size. To the best of our knowledge, this is the largest series of patients with posttreatment neck spasms in the published literature and the first to report information regarding the prevalence of this complication and its radiation dose-response relationship. The limitations of this study are the major limitations of any retrospective study. We are not able to control for confounders in treatment selection or disparities in posttreatment follow-up. In addition, our patients did not undergo objective studies such as electromyography or MRI to further clarify the underlying pathophysiology of this complication. We believe that this data is interesting and hypothesis generating; however, validation in another patient cohort is warranted.

In conclusion, our data suggests a strong dose response relationship between posttreatment neck spasms and radiation doses to the SCM. Although this complication can occur late and last for many years, fortunately, these symptoms were mild for most patients.

\section{REFERENCES}

1. Feng FY, Kim HM, Lyden TH, et al. Intensity-modulated radiotherapy of head and neck cancer aiming to reduce dysphagia: early dose-effect relationships for the swallowing structures. Int J Radiat Oncol Biol Phys 2007; 68:1289-1298.

2. Van Daele DJ, Finnegan EM, Rodnitzky RL, Zhen W, McCulloch TM, Hoffman HT. Head and neck muscle spasm after radiotherapy: management with botulinum toxin A injection. Arch Otolaryngol Head Neck Surg 2002; 128:956-959.

3. Gelblum D, Wolden S, Schupak K, Lee N. Neck spasms as a late effect of intensity modulated radiation therapy (IMRT) for head and neck cancer. Available at: http://astro2011.abstractsnet.com/pdfs/2708.pdf. Accessed February 2, 2013.

4. Stubblefield MD. Radiation fibrosis syndrome: neuromuscular and musculoskeletal complications in cancer survivors. $P M R$ 2011;3:1041-1054.

5. Custodio CM. Neuromuscular complications of cancer and cancer treatments. Phys Med Rehabil Clin N Am 2008;19:27-45.

6. Stubblefield MD, Levine A, Custodio CM, Fitzpatrick T. The role of botulinum toxin type $\mathrm{A}$ in the radiation fibrosis syndrome: a preliminary report. Arch Phys Med Rehabil 2008;89:417-421.

7. Gillette EL, Mahler PA, Powers BE, Gillette SM, Vujaskovic Z. Late radiation injury to muscle and peripheral nerves. Int J Radiat Oncol Biol Phys 1995;31:1309-1318.

8. Fajardo LF. Pathology of radiation injury. New York, NY: Masson Publishing; 1982. pp 375-377.

9. Stinson SF, DeLaney TF, Greenberg J, et al. Acute and long-term effects on limb function of combined modality limb sparing therapy for extremity soft tissue sarcoma. Int J Radiat Oncol Biol Phys 1991;21:1493-1499. 